\title{
CRRP: A Cooperative Relay Routing Protocol for IoT Networks
}

\author{
Jingwen Bai*, Yan Sun*, and Chris Phillips* \\ ${ }^{*}$ School of Electrical Engineering and Computer Science, Queen Mary University of London \\ Email: \{j.bai, yan.sun, chris.i.phillips\}@qmul.ac.uk
}

\begin{abstract}
Wireless communication is increasing along with improvements in chipset performance. New applications associated with the Internet of Things (IoT) often involve self-organization and collaboration among many autonomous wireless devices for which traditional infrastructure-based wireless networks are not suitable. Mobile Ad hoc Networks (MANETs), with their infrastructure-less, spontaneous and arbitrary multi-hop features are considered a popular approach for such scenarios, although they also face certain inherent challenges. These include: the limited communication range of each mobile node, power supply restrictions and frequent link breaks due to node movements. In this paper, in order to address the lack of a systematic cooperative routing scheme, we propose a novel protocol called Cooperative Relay Routing Protocol (CRRP) for data transmission in MANETs. Since node mobility can cause frequent radio link breaks, one of CRRP's pivotal design features is to enhance resilience, therefore improving the overall network throughput. This is achieved by forming localised cooperative (COP) topologies where appropriate. Furthermore, as nodes are often battery-powered, CRRP reduces the energy consumption when packets are forwarded by exploiting cooperative diversity. We assess the performance of CRRP via simulations and the results confirm its favorable operation compared to both classic and newer energy-aware MANET routing protocols.
\end{abstract}

\section{INTRODUCTION}

Currently, most wireless communication is based on cellular and Wireless Local Area Networks (WLANs). However, these are effectively point-to-point in nature and provide "last hop" communication between a mobile device and a wired infrastructure. Recent wireless applications can involve and benefit from machine-to-machine self-organized communication. For example, portable mobile devices in IoT can act as smart sensors, relays and data-processing units, providing distributed intelligent services. This requires the construction and maintenance of a communication network without any outside support, in spite of random device mobility. These conditions are unsuitable for current infrastructure-based cellular / WLAN networks. Mobile Ad hoc Networks (MANET) [1], with infrastructure-less, spontaneous and arbitrary multihop features are recognized as a promising solution for these scenarios. However, MANETs also face certain inherent challenges: the limited communication range of each mobile node, the restricted power supply and the possibility of link breaks due to frequent node movements. Many studies have been carried out in recent years to address these difficulties from different perspectives. Among these, cooperative communication has received much attention due to its perceived benefits, such as lower power consumption, reduced interference and potential channel diversity gain.

Alongside more mature Physical layer [2] and MAC layer [3] mechanisms to support cooperative communication, research interest has grown regarding cooperative communication in the Network layer. For example, based on the "Typical Model for Cooperative Communication" framework proposed in [4], the authors in [5] provide a systematic strategy for evaluating the cooperative routing schemes and comparing their pros and cons. In [6], the authors focus on the impact of cooperative routing and propose a routing scheme called Energy-Balanced Cooperative Routing (EBCR). Even so, little attention has been paid to how the routing topology is formed via cooperative route discovery, route confirmation and route enhancement etc. [7] targets this remaining problem and proposes a Network layer scheme called Cooperative Opportunistic Routing in Mobile Ad hoc Network (CORMAN). It employs large-scale live updates of a forwarding list together with small-scale retransmission of missing packets. Finally, compared to AODV [8], which is a widely adopted routing protocol in MANETs, some improvement is possible in terms of packet delivery ratio and latency. However, proactive source routing schemes like OLSR [9] need to consume large amounts of power to maintain up-to-date routing information about every node in the network. In order to provide efficient communication in a MANET, a reactive Network layer routing scheme, including mechanisms for cooperative route discovery, route confirmation and route enhancement, is desirable. Therefore, we propose a reactive scheme, called Cooperative Relay Routing Protocol (CRRP), which provides economic energy consumption together with robustness against mobility induced link breaks. The key contributions of this work are:

- An innovative COP Possibility Detection Algorithm for four-node COP topology creation and maintenance via a Cooperative Neighbour Table.

- A locally self-managed scheme for cooperative communication within in COP topology via a COP Table.

- A complete route discovery procedure with cooperative communication.

- A route enhancement mechanism for improved link-break resilience via a Relay Table.

- A resilience-aware route selection algorithm.

Section II of the paper gives details of the CRRP design. The scheme is then evaluated in Section III and finally conclusions 


\begin{tabular}{|c|c|c|c|c|c|}
\hline $\begin{array}{l}\text { Neighbor } \\
\text { Addr }\end{array}$ & $\begin{array}{l}\text { NSN Addr } \\
\text { List }\end{array}$ & $\mathrm{B} / \mathrm{U}$ & $\begin{array}{l}\text { Neighbor } \\
\text { Addr }\end{array}$ & $\begin{array}{l}\text { NSN Addr } \\
\text { List }\end{array}$ & $\mathrm{B} / \mathrm{U}$ \\
\hline IN3 & Src & B & IN5 & Dest & B \\
\hline $\mathrm{a}$ & & B & b & & B \\
\hline IN2 & IN5 b & B & IN1 & IN3 a & B \\
\hline
\end{tabular}

Fig. 1. Cooperative Neighbor Table Creation

are drawn in Section IV.

\section{CRRP DESIGN}

In general, CRRP (Cooperative Relay Routing Protocol) is a reactive routing protocol with proactive local enhancements for MANETs. It is reactive in the sense that a route is built only when data needs to be sent and proactive in the sense that the cooperative (COP) topology is set up in advance for all source-destinations within the network and can be locally selfmanaged. Details are introduced step-by-step in the following subsections.

\section{A. Neighbour Discovery}

1) Cooperative Neighbour Table Creation: Once a node receives a CHLO (Cooperative Hello) packet from its neighbour, the Cooperative Neighbour Table can be built based on the collected information as shown in Figure 1. Two new items are appended in the Cooperative Neighbour Table compared with the traditional Neighbor Table in AODV: the $N S N$ Addr List field and the $B / U$ field. Each neighbour's neighbours are attached to the corresponding NSN Addr List field, which facilitates building the cooperative (COP) table and maintaining the COP topology. B/U marks whether an incoming CHLO, which updates a given entry, was received via a broadcast or unicast packet. As with AODV, broadcasting is the common transmission method for its hello packets, whilst unicast is only employed by cooperative nodes and relay nodes when the COP and Relay Tables are being created in CRRP. Further details are provided in the Route Enhancement subsection.

2) COP Table Creation: If a node learns through its Cooperative Neighbour Table (with the help of the Neighbor Addr and NSN Addr List fields) that there exist two neighbor nodes that are also a common neighbour to another node via COP Possibility Detection Algorithm, shown below, a fournode COP topology is formed as illustrated in Figure 2. CRRP is proactive and self-managed in terms of COP topology, which means the COP Table, used to maintain COP topology, can be constructed based only on the Cooperative Neighbour Table instead of route establishment. The four Intermediate Nodes (INs) have specific roles in the COP topology (We use term IN to label all the nodes between the Src and the Dest along the route, as in [10]). The COP Source (Src), as the instigator of the COP topology decides the role assignment and initiates the local COP Table creation. The specific proactive principle is as follows: along the route, the first node within the COP topology that receives valid data will be regarded as the COP Src and the other Intermediate Nodes (INs) will be

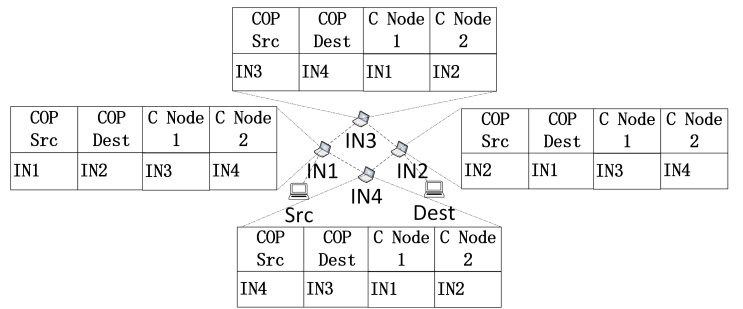

Fig. 2. COP Table Creation

assigned roles according to the COP Table of the COP Src via a Cooperative Confirm (CCON) packet. More precisely, before the COP Src forwards the data, it chooses a suitable entry from its COP Table list and places this entry in a CCON packet which will be used to notify the appropriate Cooperative (C) nodes to activate to transmit the data cooperatively and the selected COP Destination (Dest) to combine the cooperative data signals. After the COP Table is confirmed across the four INs in the COP topology, the data forwarding procedure commences, similar to the "Typical Model for Cooperative Communication" in [4]. First, the COP Src sends the data in the "normal" manner, meaning the traditional way as transmitted in [8]. However, in CRRP, there are two additional ways to send data: the "Cooperative" manner via the $\mathrm{C}$ nodes identified in the COP Table and the "Relay" manner via the relay node in Relay Table. With cooperative forwarding we exploit the overhearing transmission feature of wireless communication; i.e. when two activated $\mathrm{C}$ nodes hear the data packet transmission they cooperatively beam-form this data to the appropriate COP Dest. This reduces their individual power consumption via cooperative diversity.

The above procedure may be repeated such that the COP Dest can play the role of a COP Src in a subsequent COP topology along the route, if the node distribution permits this. Otherwise, the data will be forwarded normally. Sometimes, there are several COP topologies between two hops along a route. In this case only the COP topology activated via the COP Table will participate in cooperative communication and the others remain "silent". The CCON packet will not be sent again to trigger activation of a "silent" COP topology until the previously activated one ceases. A four-node COP topology is employed because the lower layer mechanism for this form of cooperative transmission is well understood, whilst the technological challenges regarding frame synchronization for cooperative communication are lessened. Furthermore, this approach is not restrictive, as many four-node COP topologies can coexist within the MANET. This provides ample opportunity to save energy and improve robustness.

3) Relay Table Creation: When the Cooperative Neighbour Table is updated by a CHLO packet, every IN runs the COP Possibility Detection Algorithm to update the COP Table or delete invalid entries if the COP topology no longer exists. If an entry is deleted from the COP Table but the C nodes in this entry remain neighbors, a Relay Table will be built as shown in Figure 3, which can be used to provide a data relay function between $\mathrm{C}$ nodes 1 and 2 to avoid a link break. Further details 

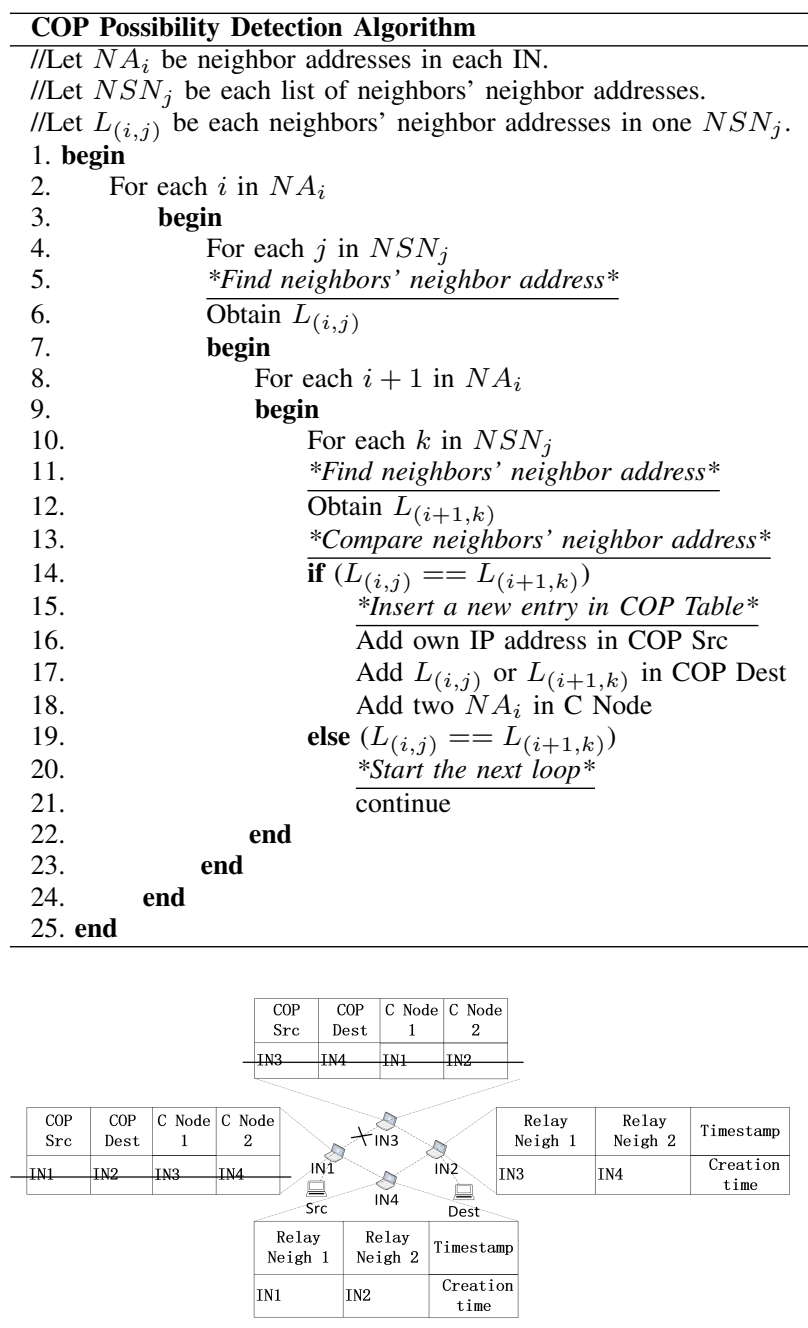

Fig. 3. Relay Table Creation

are given in the Route Enhancement section.

\section{B. Route Discovery}

When a node requires a route to a destination it must broadcast a Cooperative Route Request (CREQ) packet to seek a route across the MANET. A new CREQ packet handling procedure (including broadcasting across normal and COP topologies) is designed to allow COP topology information, if any, to be carried in a CREQ packet to the destination therefore contributing to the final route selection strategy. If more COP topologies are involved in the final route, this leads to greater robustness against mobility and improved energy savings through cooperative transmissions. As CREQ handling in a normal topology is similar to AODV, we focus here on the COP topology case. In the COP topology, once an IN receives a CREQ packet and finds that the immediate upstream node of the last hop is the COP Dest in its COP Table, it performs "last hop replacement"; that is the IN replaces last hops IP address in the CREQ packet IP list with its own IP address before rebroadcasting it to its neighbours which can make the location of COP Dest closer to the destination and reduce the total hops in the final route. Furthermore, the "last hop replacement" leads to the $\mathrm{C}$ nodes invisibility if this COP topology is selected in the final route. The invisibility of $\mathrm{C}$ nodes actually results in a virtual point-to-point connection diagonally within the COP topology even though COP Src and COP Dest may not be within each other's direct transmission range. This virtual point-to-point connection does not only contribute to saving energy via cooperative diversity, but improves the robustness against mobility. This is because if any one of the $\mathrm{C}$ nodes moves away from the COP topology, a Relay Table will be built to maintain the connection between COP Src and COP dest.

\section{Route Enhancement}

Like [8], the link is regarded as broken if one neighbor is no longer valid in Neighbor Table and this unavailable neighbor is the next hop of a valid route entry in Route Table. However in CRRP, the CHLO unicast scheme can enhance the neighbor relationship between COP Src and COP dest in the COP Table and relay neighbors in the Relay Table, which finally contributes to improve the stability of Route Table. More precisely, neighbors established by both broadcast and unicast CHLO can be used to verify the next hop validity in the Route Table and increase the possibility of a valid route being identified. The CHLO unicast scheme operates as follows: When one node receives a CHLO packet from one of its C nodes in the COP Table or relay neighbor in the Relay Table, it unicasts this CHLO to the other C node or relay neighbor. In order to utilize this enhanced neighbor relationship during data transmission to improve route robustness, a route enhancement relay principle is employed via the Relay Table. Once a COP table turns into a relay table, data will be relayed from one relay neighbor to the other in relay manner only when no direct connectivity exists between these two relay neighbors. Figure 4 provides more detail about route enhancement. Scenario (a) assumes that there is no direct connectivity between the COP Src and COP Dest. Only when both $\mathrm{C}$ nodes (C node1 and 2) move out of range, will the route be broken. This is because if only one $\mathrm{C}$ node leaves, the other $\mathrm{C}$ node will establish a Relay Table, which allows data to be relayed from the COP Src (now Relay Neighbor 1) to COP Dest (now Relay Neighbor 2) in relay manner, maintaining the route. Assume there is direct connectivity between the COP Src and COP Dest as indicated in scenario (b) of Figure 4. Due to mobility, if direct connectivity is lost, the link between COP Src and COP Dest can remain stable due to cooperative communication via two $\mathrm{C}$ nodes which is the same as scenario (a). Finally if only the COP Src and COP Dest are involved initially, and subsequently two $\mathrm{C}$ nodes (Joining INs) move into range a COP topology is formed as shown in scenario (c). At this moment, the enhanced performance will be the same as for scenario (b). The above three cases assume there is only one COP topology between a pair of COP Src and COP Dest. If more than one COP topology exist, as mentioned in COP Table Creation section, and an activated $\mathrm{C}$ node moves out of the current COP topology range, the link between the COP Src and COP Dest remains stable using the relay forwarding method. 


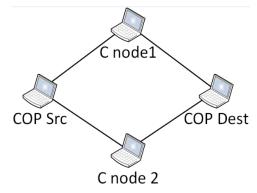

(a)

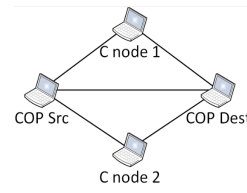

(b)

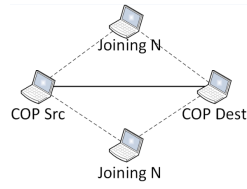

(c)

Fig. 4. Route Enhancement Scenarios

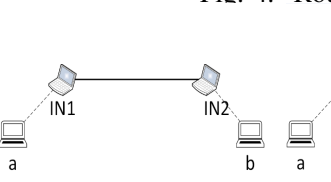

(a)

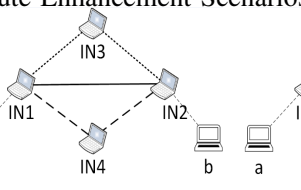

(b)

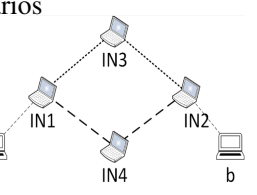

(c)
Fig. 5. COP Topology Links

If this link is itself compromised one of the silent backup COP topologies will be activated to maintain connectivity with the COP Dest.

To summarize, only when all the links between the COP Src and COP Dest are lost is the route indeed broken. Therefore, CRRP constructs a robust, energy-efficient route by employing a COP Table, Relay Table and CHLO unicast scheme.

\section{Route Selection Criteria}

In an ad hoc network, any link between two nodes can be regarded as an independent event $\varepsilon$ with outcome $\{B, \bar{B}\}$, where $\mathrm{B}$ denotes that the link breaks within a short time interval $\Delta t$ and $\bar{B}$ denotes the opposite. Assume that the link break probability is $P(B)=p$. As the link only has two states within any $\Delta t$, that is, break or non-break, so the link non-break probability is $P(B)=1-p$. Once one link along the route breaks, the route breaks. Therefore, we can calculate the route break probability according to link break probability. For CRRP, there are three types of link: a normal link (two nodes can communicate with each other directly), a connected COP link (i.e. a link exists between IN1 and IN2) and an unconnected COP link (i.e. a link does not exist between IN1 and IN2) as shown in Figure 5 from (a) to (c).

As details for calculating the link break probability of a normal link like (a) in Figure 5 have been presented in [11], we only refer to the conclusion

$$
p_{n}=p
$$

For a connected COP link like (b), we have the dotted link $L_{\text {dotted }}$, solid link $L_{\text {solid }}$ and dashed link $L_{\text {dashed }}$. For an unconnected COP link like (c), we have the dotted link $L_{\text {dotted }}$ and dashed link $L_{\text {dashed }}$. The link break probability of the dotted link, solid link and dashed link are obtained by the property of mutually exclusive events.

$$
\begin{gathered}
P\left(L_{\text {dotted }}\right)=1-\left(1-p_{n}\right)^{2}=2 p-p^{2} \\
P\left(L_{\text {solid }}\right)=p_{n}=p \\
P\left(L_{\text {dashed }}\right)=1-\left(1-p_{n}\right)^{2}=2 p-p^{2}
\end{gathered}
$$

We can then obtain the link break probabilities for the connected and unconnected COP link cases from Equation (5) and (6), respectively.

$p_{c}=P\left(L_{\text {dotted }}\right) \times P\left(L_{\text {solid }}\right) \times P\left(L_{\text {dashed }}\right)=p^{5}-4 p^{4}+4 p^{3}$

$$
p_{n c}=P\left(L_{\text {dotted }}\right) \times P\left(L_{\text {dashed }}\right)=p^{4}-4 p^{3}+4 p^{2}
$$

It is assumed that the number of normal links, connected and unconnected COP links are $n, m$, and $k$, respectively. Also, there are three types of event: the event of a normal link break $(N)$, the event of a connected COP link break (M) and the event of an unconnected COP link break (K). Therefore, according to the property of mutually exclusive events, we can obtain the route break probability of CRRP as given below

$$
P_{r_{C R R P}}=1-P(\bar{N}) \times P(\bar{M}) \times P(\bar{K})
$$

where

$$
\begin{gathered}
P(\bar{N})=1-\sum_{i=1}^{n} P_{r}(X=i)=\left(1-p_{n}\right)^{n} \\
P(\bar{M})=1-\sum_{i=1}^{m} P_{r}(X=i)=\left(1-p_{n c}\right)^{m} \\
P(\bar{K})=1-\sum_{i=1}^{k} P_{r}(X=i)=\left(1-p_{c}\right)^{k}
\end{gathered}
$$

Assuming $\varepsilon$ CREQ packets are received during CREQ_WAIT period, the sequence of CREQ packets can be denoted by $\left\{C_{p k t_{1}}, C_{p k t_{2}}, \ldots, C_{p k t_{\varepsilon}}\right\}$. A $P_{r_{C R R P}}\left(C_{p k t_{\varepsilon}}\right)$ is available according to each $C_{p k t_{\varepsilon}}$. Finally, a route with the minimum value of $P_{r_{C R R P}}$ is chosen and the corresponding $P_{r_{C R R P}}$ will be inserted into CREP before unicasting back to the source node.

$$
R_{\text {final }}=\min \left[P_{r_{C R R P}}\left(C_{p k t_{1}}\right), \ldots, P_{r_{C R R P}}\left(C_{p k t_{\varepsilon}}\right)\right]
$$

\section{Simulation And Evaluation}

\section{A. CRRP Performance Evaluation}

In order to investigate the performance of CRRP, several scenarios are explored using an OpNET simulation platform. Two classic ad hoc routing protocols are compared with CRRP to test the route robustness while a further two energy-aware routing protocols are simulated to evaluate the energy saving feature of CRRP. In order to normalize the value of the power consumption, we use the following formula

$$
P_{n}=\frac{2}{\pi} \times \arctan \left[\operatorname{con} \times e^{\left(\frac{t_{p} \times b_{t o t a l}+p p \times b_{t o t a l}}{t_{p} \times b_{\text {data }}+p p \times b_{\text {data }}}\right)}\right]
$$

The power consumption statistics consist of the processing and transmission power. $p p$ is the packet Processing Power per bit while $t_{p}$ is the Transmission Power per bit. We define the total bits transmitted in the whole network as $b_{\text {total }}$ which includes the data packet from the Application layer, control packets from the Network layer and MAC layer and the packet header of data packet added by Network layer and MAC layer. In addition, we define the data bits transmitted across the whole network as $b_{d a t a}$ which only includes the data from the Application layer. Finally, con is a selectable scaling coefficient to ensure our results are shown within a reasonable range.

\section{B. CRRP versus $A O D V$ and DSR}

Similar to the work [12], in which the authors compare cooperative and non-cooperative routing protocols to evaluate the performance, two classic non-cooperative routing protocols, AODV [8] and DSR [13], are chosen here to compare with CRRP. As with [7], AODV is chosen as one of our baselines because AODV is widely adopted and its operation is well 


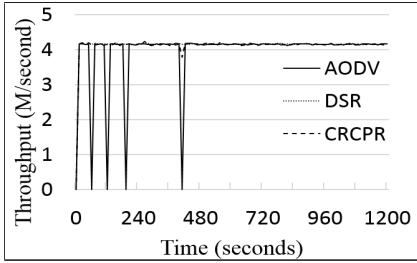

(a) 4 Mobile Nodes

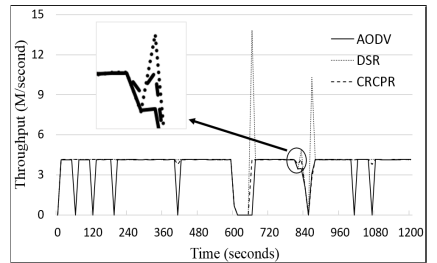

(b) 5 Mobile Nodes
Fig. 6. Typical Throughput in the 25-node Scenario with 4 / 5 Mobile Nodes

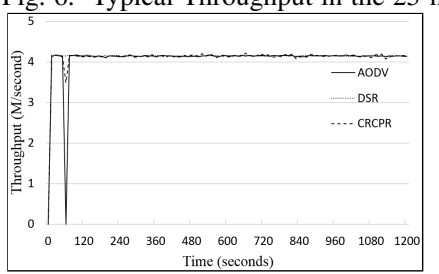

(a) 5 Mobile Nodes

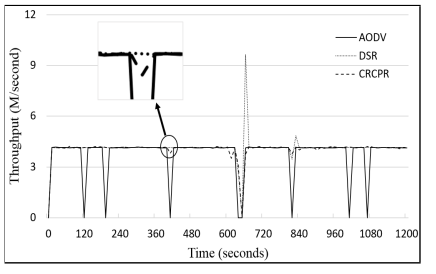

(b) 8 Mobile Nodes
Fig. 7. Typical Throughput in the 50-node Scenario with 5 / 8 Mobile Nodes

understood by the research community. Furthermore, DSR is selected as the other baseline as DSR caches back-up routes against link breaks due to mobility, which is similar to CRRP in terms of route robustness.

1) Scenario: Employing an experimental setup similar to [12], a simulation environment is configured as an area of size 1000 meters by 1000 meters. In order to estimate the link break probability we employ the same Random Walk Mobility Model as proposed in [11]. The random trajectories are recorded for each node providing repeatability to ensure comparisons are fair. As a typical ad hoc network comprises less than 100 nodes [12] [7], two network sizes are considered: 25-node and 50-node. In the 25-node scenario, we have one call with the number of random mobile nodes increasing from one to five. In the 50-node scenario, two simultaneous calls are set up with the number of random mobile nodes increasing from one to eight. The speed distribution for each mobile node in both scenarios is uniform $[0,10](\mathrm{m} / \mathrm{s})$. Each scenario runs for 20 minutes simulation time with 10 random seeds to avoid the influence of correlation effects.

2) Results:

\section{- Throughput}

All the throughput data shows instantaneous values, which can illustrate the link breaks more clearly. (a) and (b) in Figure 6 show the throughput at the destination with four and five mobile nodes in the 25-node scenario. Figure 7 shows the throughput with five and eight mobiles in the 50-node scenario.

When there are fewer than 5 mobile nodes, as with (a) in Figure 6, AODV suffers link breaks, shown as sudden drops in throughput, and has to re-establish a route for transmission. As DSR has cached routes, it just changes to the back-up route and does not need to conduct route discovery again. CRRP uses the route enhancement relay feature, so the movement only changes the transmission from the COP mode to Relay mode without suffering any link break when first three link breaks happen. The small drop for CRRP at the fourth link break of AODV is explained in (b). Once the number of mobile nodes increases to five, the link breaks happen more frequently,

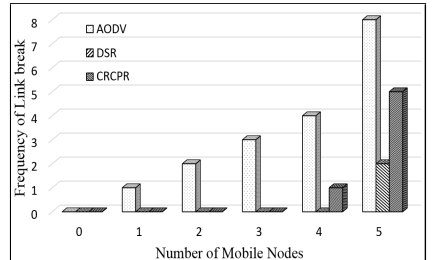

(a) 25-node Scenario

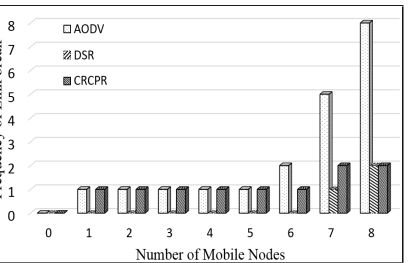

(b) 50-node Scenario
Fig. 8. Link Break Frequency

as seen in (b) in Figure 6. During the interval between 600s and 700s, all three protocols lose the route. With DSR, once the route is reconstructed, all buffered packets are sent leading to a sudden rise in throughput. Meanwhile, there is a little drop with CRRP, which is emphasized in the enlarged area. This drop indicates a link break similar to AODV, but because we employ a local repair mechanism, as in ABR [10], CRRP can restore the route more quickly and maintain high throughput. Thus, overall, CRRP performs much better than AODV in terms of link break outages and throughput. In the 50-node scenario, with mobile nodes increasing from 5 to 8 , link breaks happen more frequently as shown from (a) to (b) in Figure 7. However, CRRP has much better performance than AODV in term of reduced link breaks. With more random mobile nodes, DSR with its many cached routes performs well but requires considerable resource to maintain the back-up routes.

\section{- Link Break Frequency}

The results of (a) in Figure 8 show the link break frequency of the three protocols in the 25-node scenario. When the number of random mobile nodes increases from 1 to 3, CRRP has the same performance as DSR. With 4 and 5 random mobile nodes, DSR has the best performance due to its cached routes. However, the frequency of link breaks for CRRP remains much lower than AODV. The results for the 50-node scenario, Figure 8 (b), shows that CRRP and DSR have similar performance. However, with more mobile nodes, the back-up route scheme of DSR shows better performance. Nevertheless, in both cases of 25-node and 50-node scenario, CRRP greatly outperforms AODV.

\section{- Power Consumption}

Figure 9 shows the power consumption for both scenarios. In the 25-node case, CRRP has the best performance whilst DSR has the worst. The reason is that once a COP topology is selected for a route or a COP topology is formed locally during the transmission, the power saved will be more than $40 \%$ relative to the non-cooperative transmission case according to [4]. DSR consumes more energy due to the retransmission mechanism. For the 50-node scenario, with more nodes in the area, there are more opportunities for COP topology-based route establishment. Therefore, more energy can be saved by cooperative communication.

\section{CRRP versus MMBCR and $L P R$}

MMBCR [14] adopts lifetime prediction to avoid routes through nodes that may have low battery charge. More specifically, it selects a route with lowest route lifetime constraint, i.e. it has the highest residual energy of all possible routes 


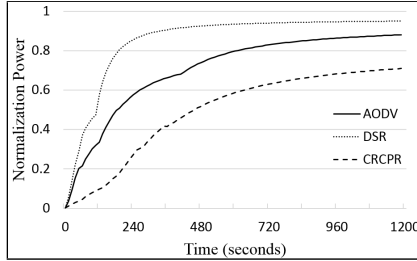

(a) 25-node Scenario

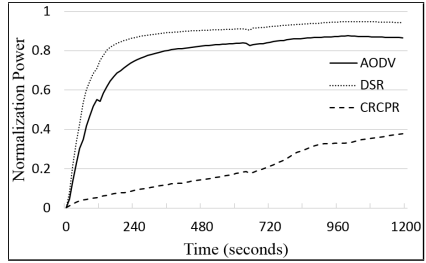

(b) 50-node Scenario
Fig. 9. Power Consumption

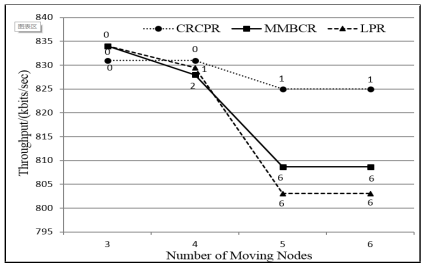

(a) Throughput

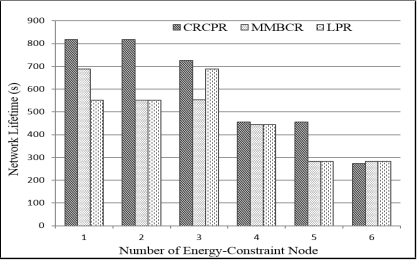

(b) Lifetime
Fig. 10. Results in the 35-node Scenario

during the route discovery period. In LPR [15], the energy drain rate is used to estimate the lifetime of the nodes. It then chooses a route with highest lifetime. Overall, both of these energy-aware routing protocols employ a similar approach, i.e. considering energy when routing to maximize the whole network lifetime.

1) Scenario: Two simulation scenarios are employed with 35 nodes randomly distributed to compare CRRP, MMBCR and LPR protocols. In the first scenario, the number of moving nodes increases from 3 to 6 and in the second scenario, the number of energy-constrained nodes is set from 1 to 6 . In both scenarios, one call is set up. Ten different seeds are tested for each scenario and the average results are illustrated below.

2) Results:

- Throughput

For MMBCR and LPR, neither have any mechanism to compensate for node mobility. Therefore, the throughput of MMBCR and LPR decreases greatly when the number of moving nodes increases, as in (a) of Figure 10. Conversely, CRRP utilizes the route enhancement relay feature to improve the robustness against mobility and thus demonstrates better throughput performance.

\section{- Lifetime}

The network lifetime is defined as the network duration when the first node along the route runs out of battery power. Both MMBCR and LPR choose routes that avoid energyconstrained nodes, where possible. Therefore, MMBCR and LPR have a better performance in certain circumstances as shown in (b) of Figure 10. However, for CRRP, the COP topologies along the route can utilize cooperative diversity to save transmission energy up to $40 \%$. Once energy-constrained nodes become involved as COP topology $\mathrm{C}$ nodes, CRRP shows the best performance.

\section{CONCLUSION}

In this work we have introduced a novel routing protocol called "Cooperative Relay Routing Protocol" based on cooperative communication to support emerging environments like IoT. By exploiting cooperative diversity with the help of COP Table, energy consumption during transmissions can be significantly reduced. Additionally, by employing a relay principle based on a Relay Table, CRRP provides greater robustness against node mobility induced link breaks. Our Network layer framework explicitly covers cooperative route discovery, route confirmation and route enhancement. This framework can be readily integrated with existing lower layer mechanisms to improve the performance of wireless ad hoc networks.

\section{REFERENCES}

[1] U. G. Swarnapriyaa, V. Vinodhini, S. Anthoniraj, and R. Anand, "Notice of violation of ieee publication principles auto configuration in mobile ad hoc networks," in Innovations in Emerging Technology (NCOIET), 2011 National Conference on, Feb 2011, pp. 61-66.

[2] J. N. Laneman, D. N. C. Tse, and G. W. Wornell, "Cooperative diversity in wireless networks: Efficient protocols and outage behavior,' IEEE Transactions on Information Theory, pp. 3062-3080, Dec 2004.

[3] H. Adam, W. Elmenreich, C. Bettstetter, and S. M. Senouci, "Core-mac: A mac-protocol for cooperative relaying in wireless networks," in Global Telecommunications Conference. GLOBECOM, Nov 2009, pp. 1-6.

[4] R. Madan, N. B. Mehta, A. F. Molisch, and J. Zhang, "Energyefficient decentralized cooperative routing in wireless networks," IEEE Transactions on Automatic Control, pp. 512-527, March 2009.

[5] Y. Wang, X. Yang, S. Yang, W. Yu, S. Bhattarai, D. Shen, and G. Chen, "Towards energy-efficient cooperative routing algorithms in wireless networks," in Consumer Communications and Networking Conference (CCNC), 2013 IEEE, Jan 2013, pp. 79-84.

[6] S. Chen, M. Huang, Y. Li, Y. Zhu, and Y. Wang, "Energy-balanced cooperative routing in multihop wireless ad hoc networks," in Соттиnications (ICC), 2012 IEEE International Conference on, June 2012, pp. 307-311.

[7] Z. Wang, Y. Chen, and C. Li, "Corman: A novel cooperative opportunistic routing scheme in mobile ad hoc networks," IEEE Journal on Selected Areas in Communications, pp. 289-296, Feb 2012.

[8] C. E. Perkins and E. M. Royer, "Ad-hoc on-demand distance vector routing," in Proceedings of the Second IEEE Workshop on Mobile Computer Systems and Applications, ser. WMCSA '99. Washington, DC, USA: IEEE Computer Society, 1999, pp. 90-. [Online]. Available: http://dl.acm.org/citation.cfm?id=520551.837511

[9] P. Jacquet, P. Muhlethaler, T. Clausen, A. Laouiti, A. Qayyum, and L. Viennot, "Optimized link state routing protocol for ad hoc networks," in Multi Topic Conference, 2001. IEEE INMIC 2001. Technology for the 21st Century. Proceedings. IEEE International, 2001, pp. 62-68.

[10] C.-K. Toh, "Associativity-based routing for ad hoc mobile networks," Wirel. Pers. Commun., vol. 4, no. 2, pp. 103-139, Mar. 1997. [Online] Available: http://dx.doi.org/10.1023/A:1008812928561

[11] L. Gong, Y. Bai, M. Chen, and D. Qian, "Link availability prediction in ad hoc networks," in Parallel and Distributed Systems, 2008. ICPADS '08. 14th IEEE International Conference on, Dec 2008, pp. 423-428.

[12] Y. Liu, J. Zhang, J. Song, R. Xu, and Z. Chen, "An ad hoc cooperative routing algorithm based on symmetric link selection," in Communication Technology (ICCT), 2010 12th IEEE International Conference on, Nov 2010, pp. 1156-1159.

[13] D. B. Johnson, D. A. Maltz, and J. Broch, "Dsr: The dynamic source routing protocol for multi-hop wireless ad hoc networks," in In Ad Hoc Networking, edited by Charles E. Perkins, Chapter 5. Addison-Wesley, 2001, pp. 139-172.

[14] C. K. Toh, "Maximum battery life routing to support ubiquitous mobile computing in wireless ad hoc networks," IEEE Communications Magazine, vol. 39, no. 6, pp. 138-147, Jun 2001.

[15] M. Maleki, K. Dantu, and M. Pedram, "Lifetime prediction routing in mobile ad hoc networks," in Wireless Communications and Networking. WCNC 2003. IEEE, vol. 2, March 2003, pp. 1185-1190 vol.2. 